\title{
Rapid Simulation of Elemental Maps in Core-Loss Electron Energy Loss Spectroscopy
}

\author{
H. G. Brown ${ }^{1 *}$, S. D. Findlay ${ }^{2}$, L. J. Allen ${ }^{3}$, J. Ciston ${ }^{1}$ and C. Ophus ${ }^{1}$ \\ 1. National Centre for Electron Microscopy (NCEM), Molecular Foundry, Lawrence Berkeley National \\ Laboratory, Berkeley, USA \\ 2. School of Physics and Astronomy, Monash University, Melbourne, Australia \\ 3. School of Physics, the University of Melbourne, Melbourne, Australia \\ * Corresponding author: hamishbrown@lbl.gov
}

Recent advances in monochromator technology have lowered the energy resolution of modern electron energy loss spectroscopy (EELS) instruments to just a few meV, opening up low energy-loss processes such as phonons and molecular vibrations as new fields of study in TEM spectroscopy [1]. These advances in monochromator technology have the potential to enable better probing of subtle bonding effects using the fine structure in the energy loss spectrum for a core-shell ionization event. This endeavour typically requires accurate accompanying image simulations (most often using the multislice method) to account for the strong elastic scattering of the electron probe.

Accurate simulation of STEM EELS images requires calculating the elastic scattering of the electron probe before and after exciting a core loss event [2]. There can be hundreds of these events, even in thin TEM samples. This is incredibly time consuming and adequately converged calculations can take up to a week even for simple crystal systems using GPU accelerated multislice implementations. In this talk we discuss the application of a recent innovation in TEM image simulation, dubbed the planewave reciprocal-space interpolated scattering matrix (PRISM) method, to the problem [3]. The PRISM method can accelerate STEM image simulation in two ways. Traditional multislice simulations directly calculate scattering of an electron probe whilst the PRISM method calculates a matrix operator (the scattering matrix) which can then be rapidly applied to all the different probe positions of interest. The PRISM method allows the use of an "interpolation factor" wherein a sparser scattering matrix is calculated, an approximation that is especially beneficial for STEM image simulations of large unit cells.

Applying the PRISM method to the problem of STEM-EELS simulation we calculate two scattering matrices: one to advance the probe to the depth of the specimen where ionization happens and a second one to then take the inelastically scattered electrons to the exit surface, this is illustrated in Fig. 1. We show that approaching the problem this way means that the calculation time only scales linearly with specimen thickness whilst the traditional multislice approach to this problem scales quadratically with thickness, see Fig. 2. The use of an interpolation factor in this context means that the calculation time scales more favourably to larger systems. We discuss progress towards an implementation of this algorithm in a way that will be accessible and useful to practitioners within the STEM-EELS community [4].

References:

[1] O. L. Krivanek, et al. Nature 514 (2014), p. 209.

[2] C. Dwyer, S. D. Findlay and L. J. Allen, Physical Review B 77 (2008), 184107.

[3] C. Ophus, Advanced Structural and Chemical Imaging 3 (2017), 13. 
[4] HGB and JC acknowledge additional funding form the DOE Early Career Research Program. Work at the Molecular Foundry was supported by the Office of Science, Office of Basic Energy Sciences, of the U.S. Department of Energy under Contract No. DE-AC02-05CH11231.

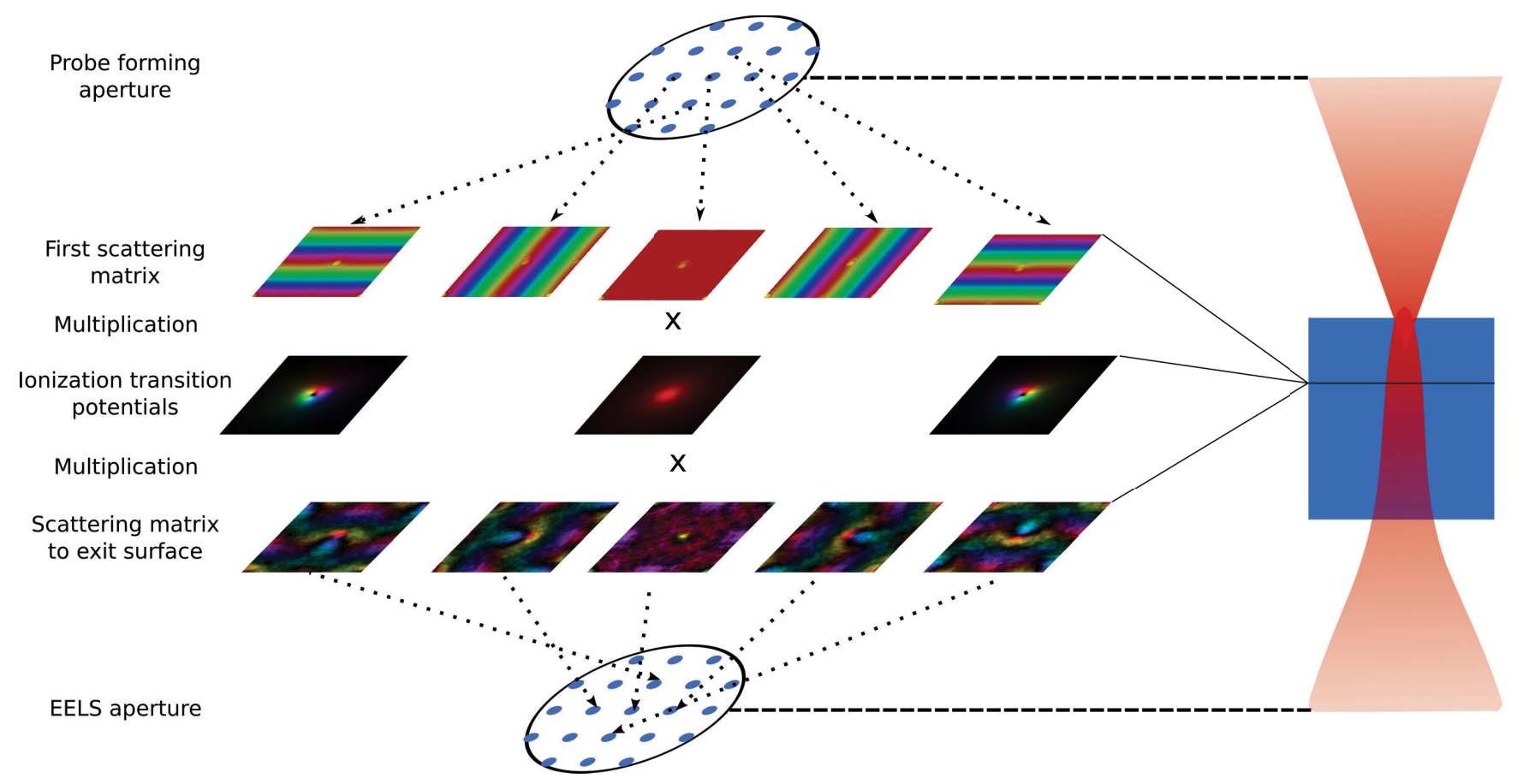

Figure 1. A sketch of the PRISM algorithm as applied to the problem of STEM-EELS simulation. One scattering matrix propagates the electron wave to the thickness at which ionization occurs, ionization is modeled through multiplication of the electron wave by a transition potential and a second scattering matrix propagates the scattered wave to the exit surface of the specimen. Storing scattering matrices typically economizes on the total number of operations that need to be performed.

(a)

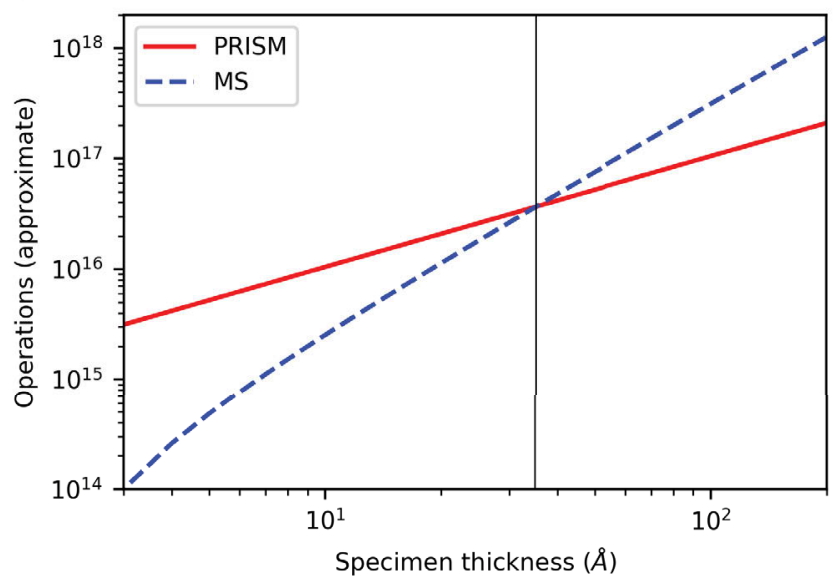

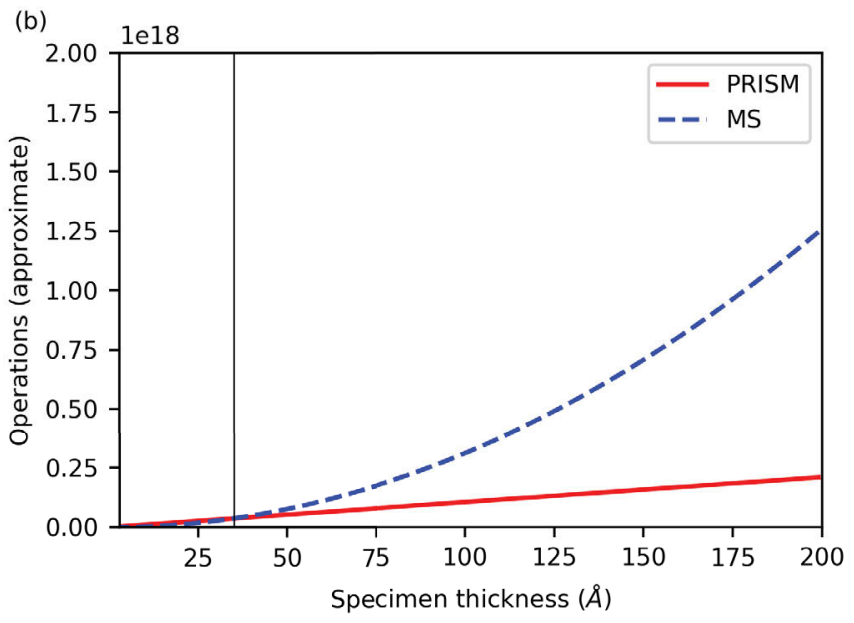

Figure 2. Comparison of the approximate numerical complexity for PRISM and a traditional multislice (MS) approaches to STEM EELS simulation as a function of specimen thickness with logarithmic (a) and linear (b) axis scales. Probe forming and EELS acceptance semi angles of $20 \mathrm{mrad}$, an accelerating voltage of $200 \mathrm{kV}, 40$ different inelastic states per slice and a simulation grid measuring $100 \AA$ by $100 \AA$ was assumed. For this case the PRISM algorithm would outperform a conventional multislice calculation for samples thicker than about $35 \AA$ (indicated by a solid vertical line). 